Pacific

Journal of

Mathematics

VIRASORO CONSTRAINTS AND HURWITZ NUMBERS THROUGH ASYMPTOTIC ANALYSIS

Yon-SEO Kim AND KefENG LiU

Volume $241 \quad$ No. 2

June 2009 


\title{
VIRASORO CONSTRAINTS AND HURWITZ NUMBERS THROUGH ASYMPTOTIC ANALYSIS
}

\author{
YON-SEO KIM AND KEFENG LiU
}

\begin{abstract}
We present a new method, using asymptotic analysis, to obtain a system of recursion relations for integrals on moduli spaces of curves. By applying the asymptotic analysis on the join-cut relation of the ELSV formula, we derive the recursion relation of $R$. Dijkgraaf and $E$. and $H$. Verlinde, which is exactly the Virasoro constraints for topological gravity. This gives an alternative proof of Witten's Conjecture using asymptotic analysis.
\end{abstract}

\section{Introduction}

We present an alternate proof of Witten's Conjecture [1991] which claims that the tautological intersections on the moduli space of stable curves $\bar{M}_{g, n}$ is governed by KdV hierarchy. M. Kontsevich [1992] first proved the conjecture by constructing combinatorial model for the intersection theory of $\bar{M}_{g, n}$ and interpreting the trivalent graph summation by a Feynman diagram expansion for a new matrix integral. A. Okounkov and R. Pandharipande [2001] and M. Mirzakhani [2007a; 2007b] (see also [Mulase and Safnuk 2006]) gave different approaches through the enumeration of branched coverings of $\mathbb{P}^{1}$ and the Weil-Petersson volume, respectively.

More recently, M. Kazarian and S. Lando [2005] obtained an algebro-geometric proof by using the ELSV formula to relate the intersection indices of $\psi$-classes to Hurwitz numbers.

Here we take an approach using asymptotic analysis applied on the join-cut relation satisfied by Hurwitz numbers. The starting point is the ELSV formula which relates the Hurwitz numbers with linear Hodge integrals

$$
H_{g, \mu}=\frac{r !}{\mid \text { Aut } \mu \mid}\left(\prod_{i=1}^{l(\mu)} \frac{\mu_{i}^{\mu_{i}}}{\mu_{i} !}\right) \int_{\bar{M}_{g, l(\mu)}} \frac{\Lambda_{g}^{\vee}(1)}{\prod_{i=1}^{l(\mu)}\left(1-\mu_{i} \psi_{i}\right)}
$$

where $r=2 g-2+l(\mu)+|\mu|$ and $\mu$ is a partition with length $l(\mu)$ and size $|\mu|=\sum_{i=1}^{l(\mu)} \mu_{i}$. Here $\psi_{i}=c_{1}\left(\mathscr{L}_{i}\right)$ where $\mathscr{L}_{i}$ is the cotangent line bundle of $i$-th marked point over $\bar{M}_{g, l(\mu)}$ and $\Lambda_{g}^{\vee}(1)=1-\lambda_{1}+\cdots+(-1)^{g} \lambda_{g}$ is the total Chern

MSC2000: primary 51P05; secondary 81R10.

Keywords: Hurwitz, Gromov, Witten, asymptotic, ELSV, Stirling, Witten conjecture, Kontsevich. 
character of dual Hodge bundle over $\overline{\mathcal{M}}_{g, l(\mu)}$. We develop asymptotic analysis on a combinatorial expression involving mapping degrees as given by partition $\mu$. Applying the asymptotic analysis on the join-cut relation of ELSV formula yields a system of relations between linear Hodge integrals. It recursively expresses each linear Hodge integral by lower-dimensional ones. We show that the first nontrivial relation of the system is the following recursion relation for the correlation functions of topological gravity [Dijkgraaf 1992]:

$$
\begin{aligned}
& \left\langle\tilde{\sigma}_{n} \prod_{k \in S} \tilde{\sigma}_{k}\right\rangle_{g}=\sum_{k \in S}(2 k+1)\left\langle\tilde{\sigma}_{n+k-1} \prod_{l \neq k} \tilde{\sigma}_{l}\right\rangle_{g}+\frac{1}{2} \sum_{a+b=n-2}\left\langle\tilde{\sigma}_{a} \tilde{\sigma}_{b} \prod_{l \in S} \tilde{\sigma}_{l}\right\rangle_{g-1} \\
& +\frac{1}{2} \sum_{\substack{S=X \cup Y \\
a+b=n-2 \\
g_{1}+g_{2}=g}}\left\langle\tilde{\sigma}_{a} \prod_{k \in X} \tilde{\sigma}_{k}\right\rangle_{g_{1}}\left\langle\tilde{\sigma}_{b} \prod_{l \in Y} \tilde{\sigma}_{l}\right\rangle_{g_{2}},
\end{aligned}
$$

which is the Virasoro constraints for topological gravity. Here $\tilde{\sigma}_{k}$ denotes the normalized gravitational descendant $(2 k+1) ! ! \psi^{k}$.

This paper is organized as follows: In Section 2, we recall the ELSV formula and the join-cut relation satisfied by Hurwitz numbers, and consequently by linear Hodge integrals. In Section 3, we develop asymptotic analysis and derive the first three relations between linear Hodge integrals. In Section 4, we show that the first nontrivial relation is precisely the Virasoro constraints in the above recursion form.

\section{ELSV formula and the join-cut relation}

It is well-known that the Hurwitz numbers satisfy the join-cut relation, and hence the linear Hodge integrals, via the celebrated ELSV formula [Ekedahl et al. 2001]

$$
H_{g, \mu}=\frac{r !}{\mid \text { Aut } \mu \mid}\left(\prod_{i=1}^{l(\mu)} \frac{\mu_{i}^{\mu_{i}}}{\mu_{i} !}\right) \int_{\overline{\mathcal{M}}_{g, l(\mu)}} \frac{\Lambda_{g}^{\vee}(1)}{\prod_{i=1}^{l(\mu)}\left(1-\mu_{i} \psi_{i}\right)} .
$$

The corresponding expressions for linear Hodge integrals in the join-cut relation of Hurwitz numbers $H_{g, \mu}$ can be understood as coming from the boundary strata of the moduli space of curves. Precisely, let us introduce the following notation:

- $\Gamma_{r}$ corresponding to a generic curve in $\bar{M}_{g, l(\mu)}$ :

$$
\Gamma_{r}=\frac{1}{\mid \text { Aut } \mu \mid} \prod_{i=1}^{n} \frac{\mu_{i}^{\mu_{i}}}{\mu_{i} !} \int_{\bar{M}_{g, n}} \frac{\Lambda_{g}^{\vee}(1)}{\prod\left(1-\mu_{i} \psi_{i}\right)}
$$

- $\Gamma_{J}^{i j}$ corresponding to the boundary stratum obtained by joining the $i$-th and $j$-th marked points for $1 \leq i \neq j \leq l(\mu)$ :

$$
\Gamma_{J}^{i j}=\frac{1}{\mid \text { Aut } \eta^{i j} \mid} \prod_{k=1}^{n-1} \frac{\eta_{k}^{i j} \eta_{k}^{i j}}{\eta_{k}^{i j} !} \int_{\overline{\mathcal{M}}_{g, n-1}} \frac{\Lambda_{g}^{\vee}(1)}{\prod\left(1-\eta_{k}^{i j} \psi_{k}\right)}, \quad \eta^{i j} \in J(\mu)
$$


where $J(\mu)$ denotes the set of join partitions and $\eta^{i j}$ is the one obtained by joining the $i$-th and $j$-th parts of $\mu$.

- $\Gamma_{C 1}^{i}(v)$ corresponding to the boundary stratum obtained by pinching around the $i$-th marked point:

$$
\Gamma_{C 1}^{i}(v)=\frac{1}{\mid \text { Aut } v \mid} \prod_{k=1}^{n+1} \frac{v_{k}^{v_{k}}}{v_{k} !} \int_{\bar{M}_{g-1, n+1}} \frac{\Lambda_{g-1}^{\vee}(1)}{\prod\left(1-v_{k} \psi_{k}\right)}, \quad v \in C_{i}(\mu),
$$

where $C_{i}(\mu)$ denotes the set of partitions obtained by cutting $\mu_{i}$ into two parts $p$ and $q$ such that $p+q=\mu_{i}$.

- $\Gamma_{C 2}^{i}\left(v_{1}, v_{2}\right)$ corresponding to the boundary stratum obtained by splitting around the $i$-th marked point:

$$
\Gamma_{C 2}^{i}\left(v_{1}, v_{2}\right)=\left(\prod_{k=1}^{n+1} \frac{v_{k}^{v_{k}}}{v_{k} !}\right) \prod_{s=1,2} \frac{1}{\mid \text { Aut } v_{s} \mid} \int_{\bar{M}_{g_{s}, n_{s}}} \frac{\Lambda_{g_{s}}^{\vee}(1)}{\prod\left(1-v_{s, k} \psi_{k}\right)}
$$

where $v_{1} \cup v_{2}=v \in C_{i}(\mu)$ with $p \in v_{1}$ and $q \in v_{2}$.

In this notation, the join-cut relation [Goulden and Jackson 1997; 1999a; 1999b; Goulden et al. 2000; Liu et al. 2003] for linear Hodge integrals, via the ELSV formula, is written as

(1) $r \Gamma_{r}=\sum_{i=1}^{n}\left(\sum_{j \neq i} \frac{\mu_{i}+\mu_{j}}{2} \Gamma_{J}^{i j}+\sum_{\nu \in C_{i}(\mu)} \frac{p\left(\mu_{i}-p\right)}{2}\left(\Gamma_{C 1}^{i, p}+\sum_{\substack{g_{1}+g_{2}=g \\ \nu_{1} \cup \nu_{2}=\nu}} \Gamma_{C 2}^{i, p}\right)\right)$.

When there's no confusion, we will denote by $\eta=\eta^{i j}$ the join-partition and by $v=v^{i, p}$ the cut-partition of splitting $\mu_{i}=p+\left(\mu_{i}-p\right)$ for some $1 \leq p<\mu_{i}$. Note that in the $\Gamma_{C 2}$-type contribution, unstable cases are included; precisely, in the cases $\bar{M}_{0,1}$ and $\bar{M}_{0,2}$, the integrals are defined to be

$$
\int_{\bar{M}_{0,1}} \frac{1}{1-k \psi_{1}}=\frac{1}{k^{2}} \quad \text { and } \quad \int_{\overline{\mathcal{M}}_{0,2}} \frac{1}{\left(1-p \psi_{1}\right)\left(1-q \psi_{2}\right)}=\frac{1}{p+q} .
$$

\section{Asymptotic analysis}

In this section, we study asymptotic behaviour of the join-cut relation (1) and obtain a system of relations between linear Hodge integrals. The expression of linear Hodge integral admits the expansion

$$
\int_{\bar{M}_{g, n}} \frac{\Lambda_{g}^{\vee}(1)}{\prod\left(1-\mu_{i} \psi_{i}\right)}=\sum_{k} \prod \mu_{i}^{k_{i}} \int_{\bar{M}_{g, n}} \prod \psi_{i}^{k_{i}}+\text { lower degree terms }
$$

with respect to the ramification degree, where $\tilde{k}=\left(k_{1}, \ldots, k_{n}\right) \in\left(\mathbb{Z}_{\geq 0}\right)^{n}$ are multiindices satisfying the constraint $\sum k_{i}=3 g-3+n$. Hence the top-degree terms 
consist of Hodge integrals of $\psi$-classes only and lower degree terms involve $\lambda$ classes. This will give a system of relations between linear Hodge integrals. More precisely, any linear Hodge integral is determined recursively in terms of either lower-dimensional or lower-degree $\lambda$-class integrals. The following asymptotic formula is crucial in asymptotic analysis.

Proposition 3.1. As $n \rightarrow \infty$, we have for $k, l \geq 0$,

$$
\begin{gathered}
e^{-n} \sum_{p+q=n} \frac{p^{p+k+1} q^{q+l+1}}{p ! q !} \longrightarrow \frac{1}{2}\left(\frac{(2 k+1) ! !(2 l+1) ! !}{2^{k+l+2}(k+l+2) !}\right) n^{k+l+2}+o\left(n^{k+l+2}\right), \\
e^{-n} \sum_{p+q=n} \frac{p^{p+k+1} q^{q-1}}{p ! q !} \longrightarrow \frac{n^{k+1 / 2}}{\sqrt{2 \pi}}-\left(\frac{(2 k+1) ! !}{2^{k+1} k !}\right) n^{k}+o\left(n^{k}\right) .
\end{gathered}
$$

Proof. Let $m \in \mathbb{N}$ be such that $1<m<n$ and consider three ranges of $p, q$ :

$$
\begin{aligned}
& R_{l}=\{(p, q) \mid p>n-m \text { and } q<m\}, \\
& R_{c}=\{(p, q) \mid m \leq p, q \leq n-m\}, \\
& R_{r}=\{(p, q) \mid p<m \text { and } q>n-m\} .
\end{aligned}
$$

Recall Stirling's formula:

$$
n !=\frac{\sqrt{2 \pi} n^{n+1 / 2}}{e^{n}}\left(1+\frac{1}{12 n}+\cdots\right)
$$

For the summation over $R_{c}$, let $m=n \epsilon$ and $p=n x$ for some $\epsilon, x \in \mathbb{R}_{>0}$ so that $m, p \in \mathbb{N}$, then we have

$$
\begin{aligned}
e^{-n} & \sum_{p=m}^{n-m} \frac{p^{p+k+1}}{p !} \frac{q^{q+l+1}}{q !}=\sum_{p=m}^{n-m} \frac{1}{2 \pi} p^{k+1 / 2} q^{l+1 / 2}(1+o(1)) \\
& \longrightarrow \frac{n^{k+l+2}}{2 \pi} \int_{\epsilon}^{1-\epsilon} x^{k+1 / 2}(1-x)^{l+1 / 2} d x+o\left(n^{k+l+2}\right) \quad \text { as } n \text { goes to } \infty \\
& =\frac{n^{k+l+2}}{2 \pi} \frac{(2 k+1) ! !(2 l+1) ! !}{(2(k+l)+3) ! !} \int_{\epsilon}^{1-\epsilon} \frac{(1-x)^{k+l+3 / 2}}{\sqrt{x}} d x+o\left(n^{k+l+2}\right)+O(\sqrt{\epsilon}) \\
& =\frac{1}{2}\left(\frac{(2 k+1) ! !(2 l+1) ! !}{2^{k+l+2}(k+l+2) !}\right) n^{k+l+2}+o\left(n^{k+l+2}\right)+O(\sqrt{\epsilon}) .
\end{aligned}
$$

As $n \longrightarrow \infty$, we can send $\epsilon \longrightarrow 0$. For the summation over $R_{l}$ and $R_{r}$, the topdegree terms belong to $O\left(n^{k+1 / 2}\right)$ and $O\left(n^{l+1 / 2}\right)$, respectively. Since we assume $k, l \geq 0$, both cases belong to $o\left(n^{k+l+2}\right)$, and this proves the first formula. For the second formula, $R_{l}$ has highest order of $n^{k+1 / 2}$ and one can show that the leading term in the asymptotic behaviour is $n^{k+1 / 2} / \sqrt{2 \pi}$. After integration by parts, $R_{c}$ 
gives the second highest term in the asymptotic behaviour

$$
\begin{aligned}
e^{-n} \sum_{p=m}^{n-1} \frac{p^{p+k+1}}{p !} \frac{q^{q-1}}{q !} & =\sum_{p=m}^{n-1} \frac{1}{2 \pi} p^{k+1 / 2} q^{l-3 / 2}(1+o(1)) \\
& \longrightarrow \frac{n^{k}}{2 \pi} \int_{\epsilon}^{1} x^{k+1 / 2}(1-x)^{-3 / 2} d x+o\left(n^{k}\right) \text { as } n \text { tends to } \infty \\
& =\frac{n^{k+1 / 2}}{\sqrt{2 \pi}}-\frac{n^{k}}{2 \pi}(2 k+1) \int_{\epsilon}^{\delta} \frac{x^{k-1 / 2}}{\sqrt{1-x}} d x+o\left(n^{k}\right) \\
& =\frac{n^{k+1 / 2}}{\sqrt{2 \pi}}-\left(\frac{(2 k+1) ! !}{2^{k+1} k !}\right) n^{k}+o\left(n^{k}\right)+O(\sqrt{\epsilon}) .
\end{aligned}
$$

This proves the second formula.

Let $\mu_{i}=N x_{i}$ for some $x_{i} \in \mathbb{R}$ and $N \in \mathbb{N}$. By taking general values of $x_{i}$, we can assume, without loss of generality, that $\mid$ Aut $\mu \mid=1$. As the ramification degree tends to infinity, that is, as $N \longrightarrow \infty$, the Hodge integral expansion (2) tends to

$$
\begin{aligned}
\prod_{i=1}^{n} \frac{\mu_{i}^{\mu_{i}+k_{i}}}{\mu_{i} !} \int_{\bar{M}_{g, n}} \prod \psi_{i}^{k_{i}}+O\left(e^{N} N^{m-1}\right) & \\
& \longrightarrow e^{|\mu|} \prod_{i=1}^{n} \frac{\mu_{i}^{k_{i}-1 / 2}}{\sqrt{2 \pi}} \int_{\bar{M}_{g, n}} \prod \psi_{i}^{k_{i}}+O\left(e^{N} N^{m-1}\right)
\end{aligned}
$$

where $m=3 g-3+n-(n / 2)$ is the highest degree of $N$ in (2). Same expansion applies to each term in (1). By taking out the common factor $e^{|\mu|}$ and applying the asymptotic formula in Proposition 3.1, we find that

$$
\begin{aligned}
& r \Gamma_{r}= N^{m+1}\left(\left(x_{1}+\cdots+x_{n}\right) \prod_{i=1}^{n} \frac{x_{i}^{k_{i}-1 / 2}}{\sqrt{2 \pi}} \int_{\overline{\mathcal{M}}_{g, n}} \prod_{i=1}^{n} \psi_{i}^{k_{i}}\right)+O\left(N^{m}\right), \\
& \Gamma_{C 1}^{i}= O\left(N^{m}\right)+\frac{N^{m+1 / 2}}{2} \sum_{k+l=k_{i}-2} \frac{(2 k+1) ! !(2 l+1) ! !}{2^{k+l+2}(k+l+2) !} x_{i}^{k+l+2} \prod_{j \neq i} \frac{x_{j}^{k_{j}-1 / 2}}{\sqrt{2 \pi}} \times \\
&\left(\int_{\overline{\mathcal{M}}_{g-1, n+1}} \psi_{1}^{k} \psi_{2}^{l} \prod_{j \neq i} \psi_{j}^{k_{j}}+\sum_{g_{1}+g_{2}=g} \int_{\overline{\mathcal{M}}_{g_{1}, n_{1}}} \psi_{1}^{k} \prod \psi_{j}^{k_{j}} \int_{\overline{\mathcal{M}}_{g_{2}, n_{2}}} \psi_{1}^{l} \prod \psi_{j}^{k_{j}}\right), \\
& \Gamma_{C 2}^{i}= N^{m+1 / 2} \prod_{j \neq i} \frac{x_{j}^{k_{j}-1 / 2}}{\sqrt{2 \pi}}\left(\sqrt{N} \frac{x_{i}^{k_{i}+1 / 2}}{\sqrt{2 \pi}} \int_{\overline{\mathcal{M}}_{g, n}} \prod_{l=1}^{n} \psi_{l}^{k_{l}}\right. \\
&\left.-\frac{\left(2 k_{i}+1\right) ! !}{2^{k_{i}+1} k_{i} !} x_{i}^{k_{i}} \int_{\overline{\mathcal{M}}_{g, n}} \prod_{l=1}^{n} \psi_{l}^{k_{l}}\right)+O\left(N^{m}\right),
\end{aligned}
$$




$$
\Gamma_{J}^{i j}=N^{m+1 / 2} \frac{\left(x_{i}+x_{j}\right)^{k_{i}+k_{j}-1 / 2}}{\sqrt{2 \pi}} \prod_{l \neq i, j} \frac{x_{l}^{k_{l}-1 / 2}}{\sqrt{2 \pi}} \int_{\bar{M}_{g, n-1}} \psi^{k_{i}+k_{j}-1} \prod_{l \neq i, j} \psi_{l}^{k_{l}}+O\left(N^{m}\right) .
$$

Putting them together in the join-cut relation (1) yields a system of relations between linear Hodge integrals as follows: First, we have a system of relations given by the spectrum of $N$-degree. Secondly, each relation given by some fixed $N$ degree stratum can be viewed as a polynomial in the $x_{i}$ 's

$$
R_{\tilde{m}}\left(x_{1}, \ldots, x_{n}\right)=\sum_{\left(s_{1}, \ldots, s_{n}\right)} C\left(s_{1}, \ldots, s_{n}\right) x_{1}^{s_{1}} \cdots x_{n}^{s_{n}}
$$

where $\tilde{m}$ is a half integer less than or equal to $m+1$ and the coefficient $C\left(s_{i}\right)$ of the homogeneous polynomial $x_{1}^{s_{1}} \cdots x_{n}^{s_{n}}$ involves linear Hodge integrals. Since the $x_{i}$ 's are independent variables, we obtain vanishing relations for each of $C\left(s_{i}\right)$ 's. In particular, the first few vanishing relations are given as follows:

- For the $N^{m+1}$-stratum, we have a trivial identity

$$
\begin{aligned}
\left(x_{1}+\cdots+x_{n}\right) \prod \frac{x_{i}^{k_{i}-1 / 2}}{\sqrt{2 \pi}} \int_{\overline{\mathcal{M}}_{g, n}} \prod \psi_{i}^{k_{i}} & \\
& -\left(x_{1}+\cdots+x_{n}\right) \prod \frac{x_{i}^{k_{i}-1 / 2}}{\sqrt{2 \pi}} \int_{\bar{M}_{g, n}} \prod \psi_{i}^{k_{i}}=0 .
\end{aligned}
$$

- For the $N^{m+1 / 2}$-stratum, we have the relation

$$
\begin{gathered}
\sum_{i=1}^{n}\left(\frac{\left(2 k_{i}+1\right) ! !}{2^{k_{i}+1} k_{i} !} x_{i}^{k_{i}} \prod_{j \neq i} \frac{x_{j}^{k_{j}-1 / 2}}{\sqrt{2 \pi}} \int_{\overline{\mathcal{M}}_{g, n}} \prod_{j}^{k_{j}}\right. \\
-\sum_{j \neq i} \frac{\left(x_{i}+x_{j}\right)^{k_{i}+k_{j}-1 / 2}}{\sqrt{2 \pi}} \prod_{l \neq i, j} \frac{x_{l}^{k_{l}-1 / 2}}{\sqrt{2 \pi}} \int_{\overline{\mathcal{M}}_{g, n-1}} \psi^{k_{i}+k_{j}-1} \prod \psi_{l}^{k_{l}} \\
-\frac{1}{2} \sum_{k+l=k_{i}-2} \frac{(2 k+1) ! !(2 l+1) ! !}{2^{k+l+2}(k+l+2) !} x_{i}^{k_{i}} \prod_{j \neq i} \frac{x_{j}^{k_{j}-1 / 2}}{\sqrt{2 \pi}}\left(\int_{\overline{\mathcal{M}}_{g-1, n+1}} \psi_{1}^{k} \psi_{2}^{l} \prod \psi_{j}^{k_{j}}\right. \\
\left.\left.+\sum_{\substack{g_{1}+g_{2}=g \\
v_{1} \cup v_{2}=v}} \int_{\overline{\mathcal{M}}_{g_{1}, n_{1}}} \psi_{1}^{k} \prod \psi_{j}^{k_{j}} \int_{\overline{\mathcal{M}}_{g_{2}, n_{2}}} \psi_{1}^{l} \prod \psi_{j}^{k_{j}}\right)\right)=0 .
\end{gathered}
$$

- Lower degree strata will give relations for Hodge integrals involving nontrivial $\lambda$-classes in terms of lower-dimensional ones. For example, the relation given by the $N^{m}$-stratum verifies the expression of $\lambda_{1}$-class

$$
12 \lambda_{1}=\kappa_{1}+\delta-\sum_{i=1}^{n} \psi_{i}
$$


where $\delta=\bar{M}_{g, \ell} \backslash \mathcal{M}_{g, \ell}$ is the boundary divisor and $\kappa_{1}=\pi_{*}\left(\psi^{2}\right)$ as the Gysin image of forgetting map $\pi_{1}: \bar{M}_{g, \ell+1} \rightarrow \bar{M}_{g, \ell}$.

\section{DVV-formula through the asymptotic analysis}

Witten's celebrated conjecture [1991] asserts that the tautological intersections on $\bar{M}_{g, n}$ are governed by the KdV hierarchy. M. Kontsevich gave the first proof [1992] by constructing proper combinatorial model for the intersection theory of $\overline{\mathcal{M}}_{g, n}$. A. Okounkov and R. Pandharipande [2001] and M. Mirzakhani [2007a; 2007b] (see also [Mulase and Safnuk 2006]) gave different approaches through the enumeration of branched coverings of $\mathbb{P}^{1}$ and the Weil-Petersson volume, respectively. More recently, M. Kazarian and S. Lando [2005] obtained an algebro-geometric proof starting from the ELSV formula [Ekedahl et al. 2001]. In this section, we show that the Virasoro constraints is encoded in the join-cut relation of the Hurwitz numbers as the first nontrivial relation among the system of relations obtained via the asymptotic analysis. Precisely we derive the recursion relation for the correlation functions of topological gravity [Dijkgraaf 1992]

$$
\begin{aligned}
\left\langle\tilde{\sigma}_{n} \prod_{k \in S} \tilde{\sigma}_{k}\right\rangle_{g}=\sum_{k \in S}(2 k+1)\left\langle\tilde{\sigma}_{n+k-1} \prod_{l \neq k} \tilde{\sigma}_{l}\right\rangle_{g}+\frac{1}{2} \sum_{a+b=n-2}\left\langle\tilde{\sigma}_{a} \tilde{\sigma}_{b} \prod_{l \in S} \tilde{\sigma}_{l}\right\rangle_{g-1} & \\
& +\frac{1}{2} \sum_{\substack{a+b=n-2 \\
S=X \cup Y \\
g_{1}+g_{2}=g}}\left\langle\tilde{\sigma}_{a} \prod_{k \in X} \tilde{\sigma}_{k}\right\rangle_{g_{1}}\left\langle\tilde{\sigma}_{b} \prod_{l \in Y} \tilde{\sigma}_{l}\right\rangle_{g_{2}} .
\end{aligned}
$$

This recursion relation consists of the Virasoro constraints written as relations for coefficients of the partition function. This recursion relation was first obtained by R. Dijkgraaf, E. Verlinde and H. Verlinde through a physical argument. In this paper, we will call formula (4) the DVV-formula. We note that similar asymptotic behaviour of Hurwitz numbers has been studied before, for example by A. Okounkov and R. Pandharipande [2001] and D. Zvonkine [2005]. They obtained the intersection numbers $\left\langle\tau_{d_{1}} \cdots \tau_{d_{p}}\right\rangle$ using asymptotics of Hurwitz numbers. In particular, A. Okounkov and R. Pandharipande [2001] showed that the Laplace transform of certain asymptotics of Hurwitz numbers is equal to Kontsevich's generating series for $\psi$ integrals:

$$
L H_{g}\left(y_{1}, \ldots, y_{l}\right)=\sum_{\sum k_{i}=3 g-3+l}\left\langle\tau_{k_{1}} \cdots \tau_{k_{l}}\right\rangle_{g} \prod_{i=1}^{l} \frac{\left(2 k_{i}-1\right) ! !}{s_{i}^{2 k_{i}+1}}, \quad s_{i}=\sqrt{2 y_{i}} .
$$

The system of relations between linear Hodge integrals that we develop in this paper can be considered as full spectrum of asymptotic relations encoded in the 
join-cut relation of Hurwitz numbers while the works of Okounkov-Pandharipande and Zvonkine concern the top-degree relation.

Now we will show that the first nontrivial relation (3) is the DVV-formula.

Theorem 1. The relation (3) is the DVV-formula (4).

Proof. Introduce formal variables $s_{i} \in \mathbb{R}_{>0}$ and recall the Laplace transformations

$$
\int_{0}^{\infty} \frac{x^{k-1 / 2}}{\sqrt{2 \pi}} e^{-x / 2 s} d x=(2 k-1) ! ! s^{k+1 / 2}, \quad \int_{0}^{\infty} x^{k} e^{-x / 2 s} d x=k !(2 s)^{k+1} .
$$

We have vanishing relations for coefficients of each monomial in (3). In particular, the term involving the monomial $x_{1}^{k_{1}} \prod_{i=2}^{n} x_{i}^{k_{i}-1 / 2}$ is given by

$$
\begin{aligned}
& 0=\left(\frac{x_{1}^{k_{1}}}{2^{k_{1}+1} k_{1} !} \prod_{i=2}^{n} \frac{x_{i}^{k_{i}-1 / 2}}{\sqrt{2 \pi}}\right) \\
& \times\left(\left(2 k_{1}+1\right) ! ! \int_{\overline{\mathcal{M}}_{g, n}} \prod_{i=1}^{n} \psi_{i}^{k_{i}}-\sum_{j \neq i} \frac{\left(2 k_{1}+2 k_{j}-1\right) ! !}{\left(2 k_{j}-1\right) ! !} \int_{\overline{\mathcal{M}}_{g, n-1}} \psi^{k_{i}+k_{j}-1} \prod \psi_{l}^{k_{l}}\right. \\
&-\frac{1}{2} \sum_{k+l=k_{1}-2}(2 k+1) ! !(2 l+1) ! !\left(\int_{\overline{\mathcal{M}}_{g-1, n+1}} \psi^{k} \psi^{l} \prod_{j=2}^{n} \psi_{j}^{k_{j}}\right. \\
&\left.\left.+\sum_{\substack{g_{1}+g_{2}=g \\
I \cup J=\{2, \ldots, n\}}} \int_{\overline{\mathcal{M}}_{g_{1}, 1+|I|}} \psi^{k} \prod_{i \in I} \psi_{i}^{k_{i}} \int_{\overline{\mathcal{M}}_{g_{2}, 1+|J|}} \psi^{l} \prod_{j \in J} \psi_{j}^{k_{j}}\right)\right) .
\end{aligned}
$$

The extra $\frac{1}{2}$ factor for the cut cases are due to different graph counting conventions. The coefficient of the join case can be obtained by $k_{1}$-fold differentiation of $\left(x_{1}+x_{j}\right)^{k_{1}+k_{j}-1 / 2}$ with respect to $x_{1}$ followed by evaluation at $x_{1}=0$. It can also be computed directly using the Laplace transformation: Applying the Laplace transformation to the above vanishing relation yields

$$
\begin{array}{r}
0=\left(\prod_{j=2}^{n}\left(2 k_{j}-1\right) ! !\right) \\
\times\left(\left(2 k_{1}+1\right) ! ! \int_{\overline{\mathcal{M}}_{g, n}} \prod_{i=1}^{n} \psi_{i}^{k_{i}}-\sum_{j \neq i} \frac{\left(2 k_{1}+2 k_{j}-1\right) ! !}{\left(2 k_{j}-1\right) ! !} \int_{\overline{\mathcal{M}}_{g, n-1}} \psi^{k_{i}+k_{j}-1} \prod \psi_{l}^{k_{l}}\right. \\
-\frac{1}{2} \sum_{k+l=k_{1}-2}(2 k+1) ! !(2 l+1) ! !\left(\int_{\overline{\mathcal{M}}_{g-1, n+1}} \psi^{k} \psi^{l} \prod_{j=2}^{n} \psi_{j}^{k_{j}}\right. \\
\left.\left.+\sum_{\substack{g_{1}+g_{2}=g \\
I \cup J=\{2, \ldots, n\}}} \int_{\overline{\mathcal{M}}_{g_{1}, 1+|I|}} \psi^{k} \prod_{i \in I} \psi_{i}^{k_{i}} \int_{\overline{\mathcal{M}}_{g_{2}, 1+|J|}} \psi^{l} \prod_{j \in J} \psi_{j}^{k_{j}}\right)\right) .
\end{array}
$$


Multiplying the above formula by a common factor $\prod_{j \neq 1}\left(2 k_{l}+1\right)$ and changing to the notation of DVV-formula given by $\tilde{\sigma}_{k}=(2 k+1) ! ! \psi^{k}$ yields the relation

$$
\begin{aligned}
\left\langle\tilde{\sigma}_{n} \prod_{k \in S} \tilde{\sigma}_{k}\right\rangle_{g}=\sum_{k \in S}(2 k+1)\left\langle\tilde{\sigma}_{n+k-1} \prod_{l \neq k} \tilde{\sigma}_{l}\right\rangle_{g} & +\frac{1}{2} \sum_{a+b=n-2}\left\langle\tilde{\sigma}_{a} \tilde{\sigma}_{b} \prod_{l \in S} \tilde{\sigma}_{l}\right\rangle_{g-1} \\
& +\frac{1}{2} \sum_{\substack{a+b=n-2 \\
S=X \cup Y, g_{1}+g_{2}=g}}\left\langle\tilde{\sigma}_{a} \prod_{k \in X} \tilde{\sigma}_{k}\right\rangle_{g_{1}}\left\langle\tilde{\sigma}_{b} \prod_{l \in Y} \tilde{\sigma}_{l}\right\rangle_{g_{2}}
\end{aligned}
$$

which is the DVV-formula (4). The factor $2 k+1$ comes from the missing $j$-th marked point in the join-graph contribution.

The DVV-formula (4) constitutes the Virasoro constraints for the point target [Dijkgraaf 1992], and hence gives another proof of Witten's Conjecture (Kontsevich's Theorem).

\section{References}

[Dijkgraaf 1992] R. Dijkgraaf, "Intersection theory, integrable hierarchies and topological field theory", pp. 95-158 in New symmetry principles in quantum field theory (Cargèse, 1991), edited by J. Fröhlich et al., NATO Adv. Sci. Inst. Ser. B Phys. 295, Plenum, New York, 1992. MR 94c:32010

[Ekedahl et al. 2001] T. Ekedahl, S. Lando, M. Shapiro, and A. Vainshtein, "Hurwitz numbers and intersections on moduli spaces of curves", Invent. Math. 146:2 (2001), 297-327. MR 2002j:14034 Zbl 1073.14041

[Goulden and Jackson 1997] I. P. Goulden and D. M. Jackson, "Transitive factorisations into transpositions and holomorphic mappings on the sphere", Proc. Amer. Math. Soc. 125:1 (1997), 51-60. MR 97j:05007 Zbl 0861.05006

[Goulden and Jackson 1999a] I. P. Goulden and D. M. Jackson, "The number of ramified coverings of the sphere by the double torus, and a general form for higher genera", J. Combin. Theory Ser. A 88:2 (1999), 259-275. MR 2000i:05010 Zbl 0936.05005

[Goulden and Jackson 1999b] I. P. Goulden and D. M. Jackson, "A proof of a conjecture for the number of ramified coverings of the sphere by the torus", J. Combin. Theory Ser. A 88:2 (1999), 246-258. MR 2000i:05009 Zbl 0939.05006

[Goulden et al. 2000] I. P. Goulden, D. M. Jackson, and A. Vainshtein, "The number of ramified coverings of the sphere by the torus and surfaces of higher genera”, Ann. Comb. 4:1 (2000), 27-46. MR 2002b:14067 Zbl 0957.58011

[Kazarian and Lando 2005] M. Kazarian and S. Lando, "An algebro-geometric proof of Witten's conjecture", MPIM-preprint 2005-55, Max-Planck-Institut, 2005, Available at http://www.mpimbonn.mpg.de/preprints/send?bid=2754.

[Kontsevich 1992] M. Kontsevich, "Intersection theory on the moduli space of curves and the matrix Airy function”, Comm. Math. Phys. 147:1 (1992), 1-23. MR 93e:32027 Zbl 0756.35081

[Liu et al. 2003] C.-C. M. Liu, K. Liu, and J. Zhou, "A proof of a conjecture of Mariño-Vafa on Hodge integrals”, J. Differential Geom. 65:2 (2003), 289-340. MR 2005m:14104 Zbl 1077.14084 [Mirzakhani 2007a] M. Mirzakhani, "Simple geodesics and Weil-Petersson volumes of moduli spaces of bordered Riemann surfaces", Invent. Math. 167:1 (2007), 179-222. MR 2007k:32016 Zbl 1125.30039 
[Mirzakhani 2007b] M. Mirzakhani, "Weil-Petersson volumes and intersection theory on the moduli space of curves", J. Amer. Math. Soc. 20:1 (2007), 1-23. MR 2007g:14029 Zbl 1120.32008

[Mulase and Safnuk 2006] M. Mulase and B. Safnuk, "Mirzakhani's recursion relations, Virasoro constraints, and the KdV hierarchy”, Preprint, 2006. arXiv math.AG/0601194

[Okounkov and Pandharipande 2001] A. Okounkov and R. Pandharipande, "Gromov-Witten theory, Hurwitz numbers, and matrix models, I”, Preprint, 2001. arXiv math.AG/0101147

[Witten 1991] E. Witten, "Two-dimensional gravity and intersection theory on moduli space", pp. 243-310 in Surveys in differential geometry (Cambridge, MA, 1990), Lehigh Univ., Bethlehem, PA, 1991. MR 93e:32028 Zbl 0757.53049

[Zvonkine 2005] D. Zvonkine, "Enumeration of ramified coverings of the sphere and 2-dimensional gravity”, Preprint, 2005. arXiv math.AG/0506248

Received April 24, 2008. Revised January 27, 2009.

\section{YON-SEO KIM}

DEPARTMENT OF MATHEMATICS

UNIVERSITY OF CHICAGO

CHICAGo, IL 60637

USA

yskim@math.uchicago.edu

http://www.math.uchicago.edu/ yskim

\section{KEFENG LIU}

CENTER OF MATH SCIENCES

ZHEJIANG UNIVERSITY

HANGZHOU, ZHEJIANG 310027

CHINA

and

Department of Mathematics

UNIVERSITY OF CALIFORNIA AT LOS ANGELES

LOS ANGELES, CA 90095-1555

USA

liu@math.ucla.edu

liu@cms.zju.edu.cn

http://www.math.ucla.edu/ liu 